\title{
Special features of the complete one-loop MSSM SUSY effects in charged and neutral semi inclusive Higgs production at LHC.
}

\section{Claudio Verzegnassi*}

Universita' di Trieste and INFN, Sezione di Trieste, Italy

E-mail: claudio@ts.infn.it

\begin{abstract}
I briefly review the status of the available one-loop corrections to the processes of top-charged Higgs and semi-inclusive bottom-neutral Higgs production in the MSSM. While the Standard Model QCD and the SUSY QCD components calculations appear to have been satisfactorily performed, the electroweak MSSM contributions have not been completely computed in the existing previous works. We performed the complete calculations of these terms and showed that their effect can be quite relevant, and rather different from that derivable from previously used simplified approximations. A satisfactory numerical description of these processes in the MSSM should therefore necessarily retain the one-loop electroweak component.
\end{abstract}

Third International Workshop on Prospects for Charged Higgs Discovery at Colliders - CHARGED2010, September 27-30, 2010

Uppsala Sweden

\footnotetext{
* Speaker.
} 


\section{Introduction}

The results that I will show have been obtained working in the 5FNS and considering the 4 semi-inclusive processes:

$$
\begin{aligned}
& b g \rightarrow b h, \quad\left(h=h^{0}, H^{0}, A^{0}, \quad \text { neutral MSSM Higgses }\right) \\
& b g \rightarrow t H^{-}, \quad\left(H^{-}=\text {charged MSSM Higgs }\right) .
\end{aligned}
$$

in the MSSM and also in the 2HDM, Type 2, approach, for $M_{H}>m_{t}$. We shall adopt the terminology of [1] for bottom production and of [2] for top production.

At the lowest tree level order, the four considered processes are described by the Feynman diagrams in Fig. (1). To understand the main goals of the theoretical efforts to be described, a good starting point is to derive the MSSM expression of the relevant (t,b H=Higgs) Yukawa Lagrangian. In the notation of [3] this reads:

$$
\begin{aligned}
-\mathscr{L}_{y u k}^{(0)}= & \left\{\frac{\sqrt{2}}{\mathrm{v}}\left[m_{b} \tan \beta \bar{t}_{L} b_{R} H^{+}+m_{t} \cot \beta \bar{b}_{L} t_{R} H^{-}\right]+h . c .\right\}+ \\
& +\frac{m_{b}}{\mathrm{v} \cos \beta}\left[\bar{b} b\left(-\sin \alpha h^{0}+\cos \alpha H^{0}\right)-i \bar{b} \gamma_{5} b A^{0} \tan \beta\right]+ \\
& +\frac{m_{t}}{\mathrm{v} \sin \beta}\left[\bar{t} t\left(\cos \alpha h^{0}+\sin \alpha H^{0}\right)+i \bar{t} \gamma_{5} t A^{0} \cos \beta\right] .
\end{aligned}
$$

In the previous expression (where I assumed $V_{t b}=1$ ), $h^{0}, H^{0}, A^{0}$ are the physical neutral CP even and odd Higgses (CP is assumed to be conserved), $H^{ \pm}$is the physical charged Higgs. In principle, there are six free parameters (the four masses and the two angles).In the MSSM at this lowest order only two parameters are independent, usually taken as $M_{A^{0}}$ and $\tan \beta$.

In a model with one more (extra SM) Higgs Doublet and two different vevs like in the MSSM (2HDM) the six parameters are free.

Looking at the LO Lagrangian one sees that taking large values (e.g. >10) of $\tan \beta$ the term proportional to $m_{t}$ in the btH interaction can be ignored.

The four processes $b g \rightarrow b h$ and $b g \rightarrow t H$ are therefore described in this regime by the same parameters, $\tan \beta, M_{A^{0}}$ and $m_{b}$. This suggests the criterion of making a common study and comparison of the four reactions, that must necessarily involve the higher order radiative corrections and, also, the poor boy small $\tan \beta$ (e.g. <10) region.

\section{One-loop calculation}

Moving to higher orders in the MSSM requires first of all a choice of the SUSY parameters description. Next, strong and EW interactions effects must be computed (in a possibly terrifying way, where a number $N \gg 1$ of infinities must be exactly cancelled) and suitable definitions for certain parameters must be adopted.

A particularly relevant example of the latter sentence is that of the choice of the bottom mass.

At the lowest order, Eq.(1.3), $m_{b}$ is taken as the pole mass. But as soon as one moves to higher orders, this definition is not always safe and other renormalization schemes are preferable, see e.g. [4]. 
In the specific MSSM case, a particolarly convenient choice for $m_{b}$ is that of the Dimensional Reduction renormalization scheme [5]. Different mixed choices are however allowed (with some care).

In the 5FNS treatment of the four considered semi-inclusive production processes one finds two types of QCD corrections, that might be called of diagrammatic and bottom pdf origin.

The latter ones originate from the (known) fact that in the 5FNS the bottom parton is described by a proper pdf that resums collinear logarithms and is already of $\mathscr{O}\left(\alpha_{s}\right)$ (see discussion and references of [1], page 2). Moving to NLO replaces the LO version (CTEQ6L) with the NLO one.

The SM and SUSY QCD NLO diagrammatic corrections for the considered associates processes in the 5FNS have been computed by a number of authors [6].

As a general feature, SM QCD corrections to the total rates are large and positive, while SUSY QCD effects are negative and sizeable for large values, e.g.>10, of $\tan \beta$. This can be relatively easily understood as a consequence of the special large $\tan \beta$ parametrization of these effects originally proposed by Carena et al., with the introduction of an effective Yukawa Lagrangian containing the glorious parameter $\Delta_{b}$.

At the origin of the formalism there is the simple observation that in the lowest order Yukawa Lagrangian the bottom quark only interacts with d-type Higgses, while at higher MSSM order an interaction of SUSY QCD origin with u-type Higgses is introduced.

Starting from this observation and after bright steps one is led to a description of the 4 processes valid in the large $\tan \beta$ range (in fact, the leading $\tan \beta$ effects are resummed to all orders) and based on an effective Yukawa Lagrangian where a modified bottom mass enters:

$$
\begin{aligned}
& \mathscr{L}_{y u k}^{(e f f) \tan \underline{\beta} \gg 1} \frac{1}{\mathrm{v}} \frac{\overline{m_{b}}(Q)}{1+\Delta_{b}} . \\
& \left\{\left[\frac{\sin \alpha}{\cos \beta}-\Delta_{b} \frac{\cos \alpha}{\sin \beta}\right] \bar{b} b h^{0}+\left[-\frac{\cos \alpha}{\cos \beta}+\Delta_{b} \frac{\sin \alpha}{\sin \beta}\right] \bar{b} b H^{0}+\right. \\
& \tan \beta i \bar{b} \gamma_{5} b A^{0}+\sqrt{2} \tan \beta\left(H^{+} \bar{t}_{L} b_{R}+H^{-} \bar{b}_{R} t_{L}\right) .
\end{aligned}
$$

The SUSY QCD contribution to $\Delta_{b}$ depends on the values of the involved MSSM parameters. For certain choices one sees that $\Delta_{b}$ can be positive, proportional to $\tan \beta$ and thus large. The denominator $\left(1+\Delta_{b}\right)$ therefore decreases the rates of the processes.

In conclusion, NLO QCD effects are known (with their related theoretical uncertainty, due to factorization and renormalization scale dependence, discussed in the quoted references).

What about NLO electroweak corrections? For the considered processes, NLO MSSM EW radiative corrections have been computed in the following ways:

Dawson et al. have used a generalized $\Delta_{b}$ approach. Beyond the gluino-sbottom diagram, there are similar SUSY EW graphs, e.g. one with chargino-stop. This can be resummed and provides a $\Delta_{b}^{E W}$ effect which is also enhanced for large $\tan \beta$ and is supposed to be the dominant SUSY EW contribution. Numerical results are given in this approximation.

Berger et al. add to the SUSY QCD $\Delta_{b}$ term other NLO supersymmetric contributions. In the (usual) large $\tan \beta$ limit, these extra MSSM non-SUSY QCD $\Delta_{b}$ effects are small and negligible. MSSM EW effects seem not to be relevant. 
The dignity of MSSM EW effects is reconsidered in the fully inclusive process of Higgs production by bottom fusion in [7].

This paper performs the complete NLO MSSM EW calculation for the process and separates the resummed extra $\Delta_{b}^{E W}$ effects (wino-higgsino-stop, wino-higgsino- sbottom,..) from the remaining ones.

The result is that the $\Delta_{b}^{E W}$ effects are well competitive with the SUSY QCD ones, and of opposite sign. The remaining ones give a smaller (relative few percent) effect.

Our conclusion is that the NLO MSSM EW effects must be accurately computed. This is what we did.

\section{NLO MSSM EW effects}

The results that I will quote are contained in a couple of papers. For top-charged Higgs calculation [8], for bottom-neutral Higgs calculation [9].

In both papers, the complete one-loop MSSM EW effects (including QED) have been computed. NLO QCD has not been included (it already exists). The resummed $\Delta_{b}^{S U S Y} Q C D$ term has been inserted in the $b h$ production processes, and omitted in the $t H$ production case.

The EW calculation is valid for all $\tan \beta$ values, including the usually neglected small ones.

For bh we chose the Dimensional Reduction Renormalization scheme, with the (kinematical) bottom mass defined in the OS system.

For tH we chose the Renormalization scheme of [10], with the b,t masses on shell. The bottom pdf has been computed in the $\overline{M S}$ system at scale $m_{t}+m_{H}$ or $m_{h}$.

We have calculated invariant mass distributions and total rates with only EW (and SUSY QCD for bh) NLO effects for different parameter space scenarios, for the MSSM and for the 2HDM.

A few illustrative Figures. In the bh process we have (also) determined the EW K factor, defined as the ratio of the computed one-loop (BORN +SUSY QCD + MSSM EW) rate to the $\left(\right.$ BORN +SUSY QCD) one. We have considered the SPP2 scenario with fixed $M_{A^{0}}=250 \mathrm{GeV}$. The other input parameters are shown in Fig. (2). The sparticle masses and mixing angles have been obtained with the code FeynHiggs. The results for $A^{0}, H^{0}, h^{0}$ production are shown in the Fig. (3)

What about tH? We have computed the purely EW (no SUSY QCD) MSSM K factor in two mSUGRA scenarios and they are shown in Fig. (4)

The EW effect is modest for $\tan \beta=10$. For $\tan \beta=50$, it reaches a relative 20 percent size (to be compared with possible 30-40 percent effects for Berger et al.)

Again, it appears that MSSM EW NLO effects can be relevant and should be fully computed. As one sees from the Figure, the NLO MSSM EW effect is modest (a few percent) for $A^{0}$ and $H^{0}$. But for $h^{0}$ it becomes large and negative (a relative 20 percent) when $\tan \beta$ approaches 40, and is of opposite sign (a relative 10 percent) and apparently increasing when $\tan \beta$ approaches 10 .

Certainly, for bh production the NLO MSSM EW effect should not be assumed to be negligible.

A reasonable question that arises at this point, having verified the possible relevance of the complete NLO EW MSSM effects, is the following one: 
Are the EW effects of non- $\Delta_{b}$ type really important? In other words, could one use the (much simpler) Improved Born Approximation (IBA) with only resummed SUSY QCD and (all) EW $\Delta_{b}$ terms, like in the Dittmaier et al. b-fusion paper, where these contributions seem to be by far the leading ones?

\section{Validity of the IBA and search of a different approximation}

To find an answer to this question, we have compared our full one-loop calculation with the mentioned IBA, keeping in both terms the full resummed SUSY QCD $\Delta_{b}$ term and allowing as in the previous example $\tan \beta$ to vary in the SPP2 scenario with $M_{A^{0}}=250 \mathrm{GeV}$. The results are shown in Fig. (5).

As one sees from the Figure, there is a rather strong difference between the complete one-loop calculation and the IBA, that reaches a relative 25 percent size for large $\tan \beta$.

In the same Figures, one sees the result of a different Reduced Vertex Approximation (RVA), that sums at one loop effects of a larger number of bottom vertices and reproduces much better the complete calculation.

So for bh, IBA seems to be in trouble. For tH the calculation of the IBA approximation is being performed in the same $\overline{D R}$ renormalization scheme.

\section{Which special information can be obtained by NLO MSSM effects?}

As a first example, we considered the ratio of the MSSM and 2HDM rates at variable $\tan \beta$ (with another parameter e.g. $M_{A^{0}}$ kept fixed).

This ratio should be SM QCD independent, and would therefore provide the size of the genuine SUSY content (including SUSY QCD) of the MSSM with reasonable accuracy.

This ratio has already been computed for tH by Berger et al. in a certain scenario computing only a part of the MSSM EW NLO with the SUSY QCD $\Delta_{b}$ term.

The 2 HDM rate is much larger for large $\tan \beta$ (almost a factor 2 for $\tan \beta=40$ ) but coincides with the MSSM when $\tan \beta$ becomes smaller.

This is what one would expect from the presence of the $\Delta_{b}$ term.

We have performed the same comparison for bh, again allowing $\tan \beta$ to vary for fixed $M_{A^{0}}$ at different values. Only the $M_{A^{0}}=250 \mathrm{GeV}$ result for $h^{0}$ is shown in Fig. (6) is shown.

As one sees, the $2 \mathrm{HDM}$ provides a rate whose ratio with the MSSM one follows the identical $\tan \beta$ behaviour in all neutral Higgs cases. For large $\tan \beta$ the $2 \mathrm{HDM}$ rate is definitely larger, more or less like in the Berger plot.

If $\tan \beta$ were known, it might be possible to identify the true Model.

This leads to the last question of my presentation: could $\tan \beta$ be measured?

\section{A new proposed measurement}

A problem to identify SUSY parameters is the presence of strong interactions that introduce in the theoretical estimate a scale uncertainty that is sometimes as large as the SUSY effect to be measured. 
On the other hand, strong interactions increase the value of the rates, which produces more statistics. So, they are clearly useful.

The situation appears to me somehow similar to the old case of LEP1 Hadron production at the $\mathrm{Z}$ resonance.

The idea was to define an observable that was independent of strong interactions [11] i.e.: The Longitudinal Polarization Asymmetry $A_{L R}\left(M_{Z}\right)$.

First question: does such an observable exist in one of the considered processes? In principle, Yes for the $\mathrm{tH}$ process (rates for $t_{L}$ and $t_{R}$ production are certainly different).

A detailed discussion of possible measurements of top polarization asymmetry from charged slepton production, suggesting the natural extension of the work to the process of $\mathrm{tH}$ production, has very recently been provided in [12].

Could a certain longitudinal polarization asymmetry be SM QCD independent (i.e. SUSY detector)?

Before tackling this problem, we computed the purely EW NLO value of this asymmetry, defined as the ratio of difference and sum of the polarized rates, starting from the previously defined tH benchmark point (SPS1) and allowing $\tan \beta$ to vary with SUSPECT. Fig. (7)shows the left-right asymmetry.

Qualitatively : one sees a potentially relevant feature in the low $\tan \beta$ region $(2<\tan \beta<10)$, with a drastic change of sign. But SUSY QCD could be also relevant, and should be computed [13].

Our hope: SM QCD might cancel in the ratio. Then $A_{L R}$ might be considered as a possible SUSY measurement in the all $\tan \beta$ region, if combined with measurements of all tH and bh rates.

\section{Conclusions}

The role of the NLO MSSM SUSY corrections appears to be relevant both at the QCD and at EW level in the production of $\mathrm{tH}$ and bh. It seems appropriate to compute the $\Delta_{b} \mathrm{QCD}$ effect , correctly resummed, independently of the complete NLO EW contribution: no separation of priviledged resummed $\Delta_{b}^{E W}$ terms and poor people remaining NLO EW ones: Democracy must win !!!
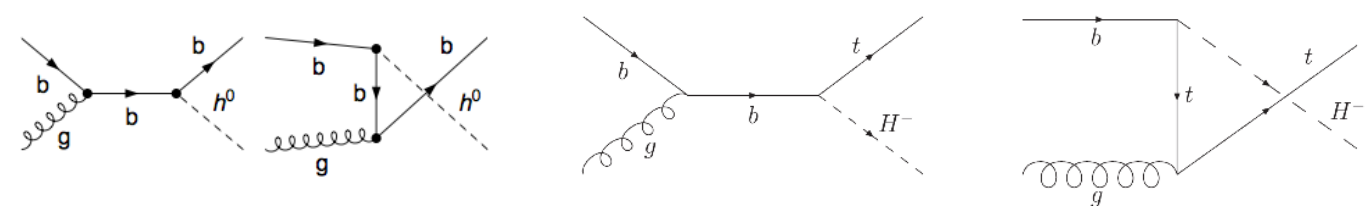

Figure 1: Born diagrams for bottom or top production.

\section{References}

[1] S. Dawson, C. B. Jackson, L. Reina and D. Wackeroth, "Higgs production in association with bottom quarks at hadron colliders," Mod. Phys. Lett. A 21, 89 (2006) [arXiv:hep-ph/0508293]. 


\begin{tabular}{c|c|c|c|c|c|c|c|c}
\hline Scenario & $\tan \beta$ & $M_{A^{0}}$ & $M_{\tilde{q}, 1}$ & $M_{\tilde{q}, 2}$ & $M_{\tilde{q}, 3}$ & $M_{1}$ & $M_{2}$ & $M_{\tilde{g}}$ \\
\hline $\mathrm{SPP}_{1}$ & 15 & 350 & 350 & 350 & 250 & 90 & 150 & 800 \\
$\mathrm{SPP}_{2}$ & variable & 250 & 500 & 500 & 400 & 90 & 200 & 800 \\
\hline
\end{tabular}

Figure 2: $S P P_{1,2}$ input parameters.

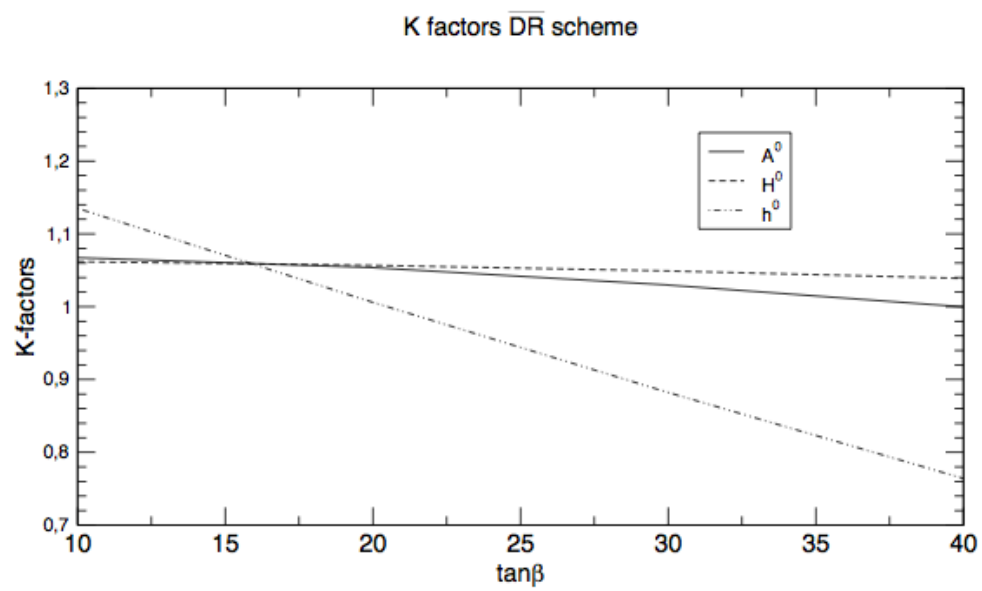

Figure 3: $K$ factor for neutral Higgs production in the MSSM.

\begin{tabular}{|c|c|cc|cc|}
\hline \multirow{2}{*}{ mSUGRA scenario } & \multirow{2}{*}{$\sigma_{\text {Born }}$} & \multicolumn{2}{|c|}{ SUSY } & \multicolumn{2}{c|}{$2 \mathrm{HDM}$} \\
\cline { 3 - 6 } & & $\sigma_{1-\text { loop }}$ & K-factor & $\sigma_{1-\text { loop }}$ & K-factor \\
\hline LS2 & 5.589 & 4.545 & 0.813 & 5.867 & 1.050 \\
SPS1a & 0.04207 & 0.04145 & 0.985 & 0.04170 & 0.991 \\
\hline
\end{tabular}

Figure 4: mSUGRA benchmark points for charged Higgs production.

[2] E. L. Berger, T. Han, J. Jiang and T. Plehn, "Associated production of a top quark and a charged Higgs boson,” Phys. Rev. D 71, 115012 (2005) [arXiv:hep-ph/0312286].

[3] M. S. Carena, D. Garcia, U. Nierste et al., "Effective Lagrangian for the anti-t b H+ interaction in the MSSM and charged Higgs phenomenology," Nucl. Phys. B577, 88-120 (2000). [hep-ph/9912516].

[4] Beneke and Singer, Phys.Lett.B471, 233 (1999).

[5] W. Siegel, Phys.Lett.B84,193(1979))

S. Heinemeyer, W. Hollik, H. Rzehak et al., "High-precision predictions for the MSSM Higgs sector at O(alpha(b) alpha(s)), Eur. Phys. J. C39, 465-481 (2005). [hep-ph/0411114]. 
[6] S-H. Zhu, "Complete next-to-leading order QCD corrections to charged Higgs boson associated production with a top quark at the CERN Large Hadron Collider“, Phys. Rev. D67, 075006 (2003);

T. Plehn, "Charged Higgs boson production in bottom-gluon fusion”, Phys. Rev. D67, 014018, (2003)

Gao, Guangping and Lu, Gongru and Xiong, Zhaohua and Yang, Jin Min, Gao,Lu,Xiong, Yang, "Loop effects and nondecoupling property of supersymmetric QCD in $g b \rightarrow t H^{-}$", Phys.Rev.D66, 015007(2002);

Campbell, J. and Ellis, R. K. and Maltoni, F. and Willenbrock, S., "Higgs-boson production in association with a single bottom quark”, Phys.Rev.D67,095002 (2003);

[7] S. Dittmaier, M. 1. Kramer, A. Muck and T. Schluter, "MSSM Higgs-boson production in bottom-quark fusion: Electroweak radiative corrections, JHEP 0703, 114 (2007) [arXiv:hep-ph/0611353].

[8] M. Beccaria, G. Macorini, L. Panizzi et al., "Associated production of charged Higgs and top at LHC: The Role of the complete electroweak supersymmetric contribution,” Phys. Rev. D80, 053011 (2009). [arXiv:0908.1332 [hep-ph]].

[9] M. Beccaria, G. O. Dovier, G. Macorini et al., "Semi-inclusive bottom-Higgs production at LHC: The complete one-loop electroweak effect in the MSSM," [arXiv:1005.0759 [hep-ph]].

[10] L. H. Wan, W. G. Ma, R. Y. Zhang and Y. Jiang, "Electroweak corrections to the charged Higgs boson decay into chargino and neutralino," Phys. Rev. D 64, 115004 (2001) [arXiv:hep-ph/0107089].

[11] B. W. Lynn and C. Verzegnassi, "LONGITUDINAL e- BEAM POLARIZATION ASYMMETRY IN e+ e- $\rightarrow$ HADRONS,” Phys. Rev. D 35, 3326 (1987).

[12] M. Arai, K. Huitu, S. K. Rai and K. Rao, "Single production of sleptons with polarized tops at the Large Hadron Collider,” JHEP 1008, 082 (2010) [arXiv:1003.4708 [hep-ph]].

[13] M. Beccaria, A. Djouadi, G. Dovier, G. Macorini, F. M. Renard, C.V., in progress. 


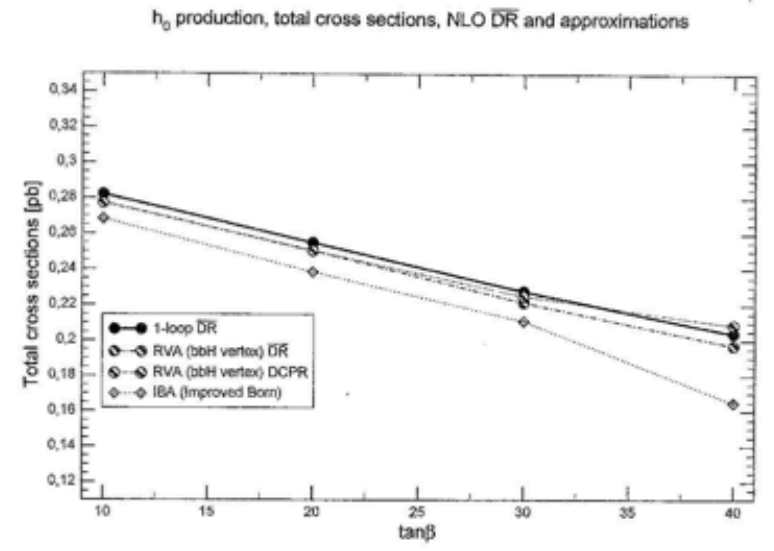

$H_{0}$ production, total cross sections, NLO $\overline{D R}$ and approximations

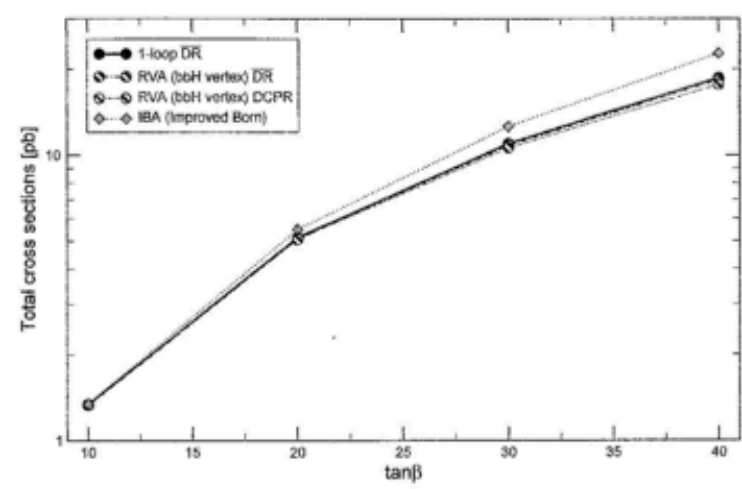

$A_{0}$ production, total cross sections, NLO $\overline{D R}$ and approximations

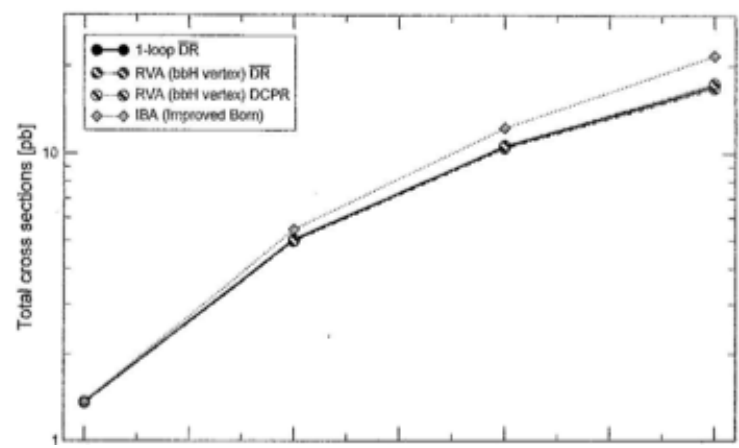

Figure 5: IBA, RVA and full one-loop for neutral Higgs production. 


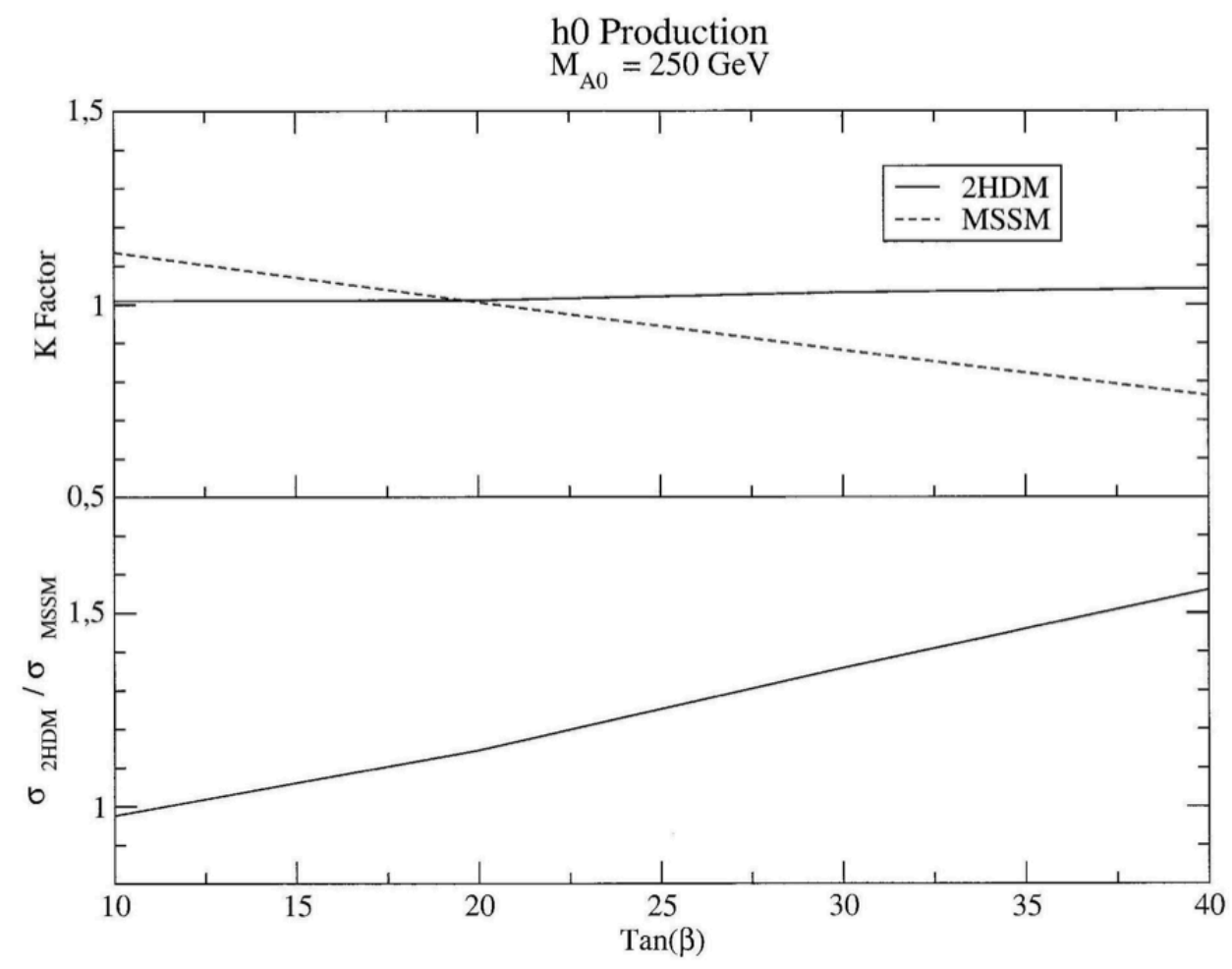

Figure 6: $2 \mathrm{HDM}$ vs MSSM for $h^{0}$ production at $M_{A^{0}}=250 \mathrm{GeV}$.

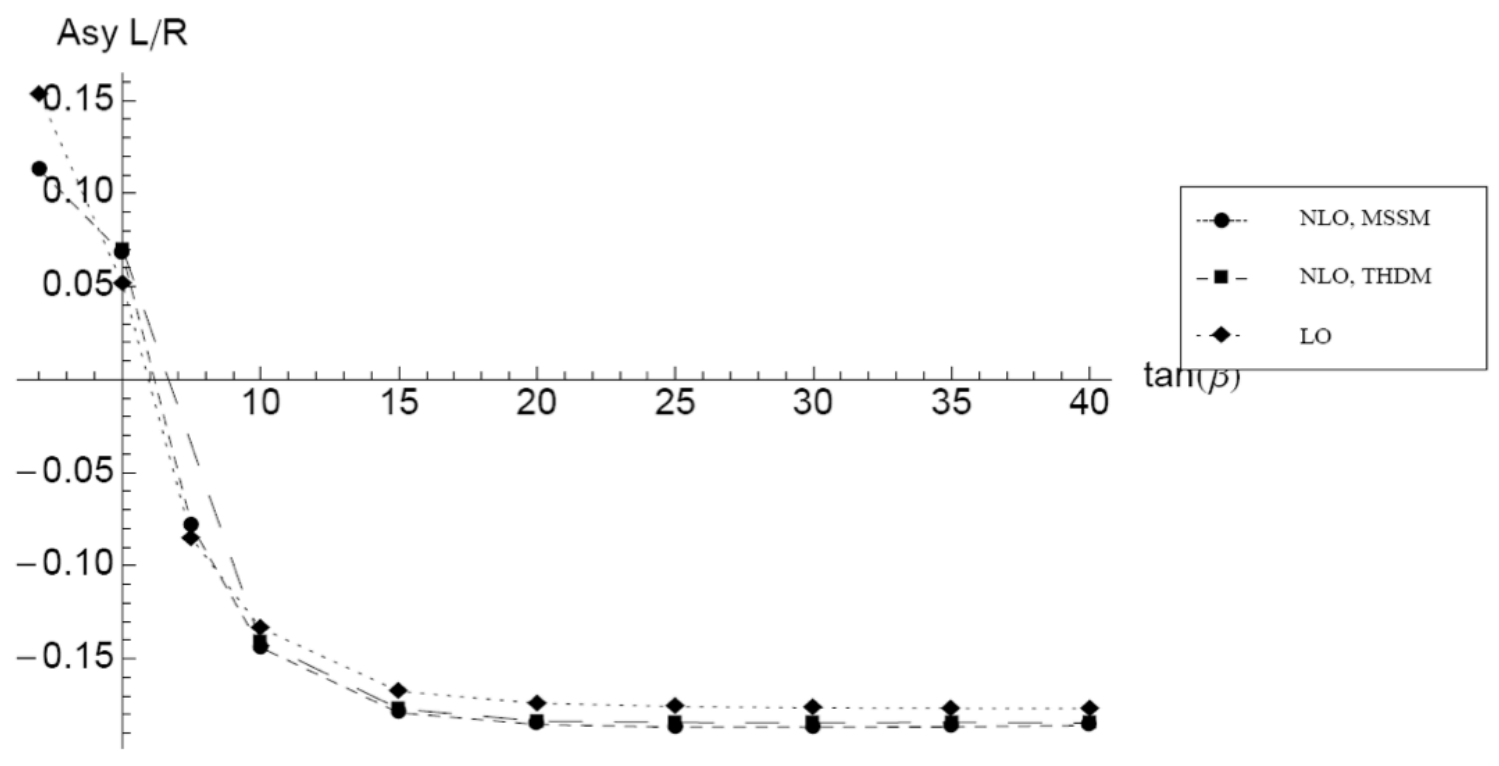

Figure 7: Top polarization asymmetry and its $\tan \beta$ dependence. 